\title{
ADHERENCE TO A SIX-DOSE REGIMEN OF ARTEMETHER-LUMEFANTRINE FOR TREATMENT OF UNCOMPLICATED PLASMODIUM FALCIPARUM MALARIA IN UGANDA
}

\author{
CAROLE FOGG, FRANCIS BAJUNIRWE, PATRICE PIOLA, SAMUEL BIRARO, FRANCESCO CHECCHI, \\ JAMES KIGULI, PROSCOVIA NAMIIRO, JOY MUSABE, AGNES KYOMUGISHA, AND JEAN-PAUL GUTHMANN \\ Epicentre, Paris, France; Médecins Sans Frontières, Paris, France; Mbarara University of Science and Technology, Mbarara, Uganda; \\ Mbarara University Teaching Hospital, Mbarara, Uganda
}

\begin{abstract}
Measuring baseline levels of adherence and identifying risk factors for non-adherence are important steps before the introduction of new antimalarials. In Mbarara in southwestern Uganda, we assessed adherence to artemetherlumefantrine $\left(\right.$ Coartem $\left.^{\circledR}\right)$ in its latest World Health Organization blister formulation. Patients with uncomplicated Plasmodium falciparum malaria were prescribed artemether-lumefantrine and received an explanation of how to take the following five doses at home. A tablet count was made and a questionnaire was completed during a home visit. Among 210 analyzable patients, $21(10.0 \%)$ were definitely or probably non-adherent, whereas 189 (90.0\%) were probably adherent. Age group was not associated with adherence. Lack of formal education was the only factor associated with non-adherence after controlling for confounders (odds ratio $=3.1,95 \%$ confidence interval [CI] $=$ 1.1-9.7). Mean lumefantrine blood levels were lower among non-adherent $(\mathrm{n}=16)(2.76 \mu \mathrm{g} / \mathrm{mL}, 95 \%$ CI $=1.06-4.45)$ than among adherent $(\mathrm{n}=171)(3.19 \mu \mathrm{g} / \mathrm{mL}, 95 \% \mathrm{CI}=2.84-3.54)$ patients, but this difference was not statistically significant. The high adherence to artemether-lumefantrine found in our study suggest that this drug is likely to be very effective in Mbarara provided that patients receive clear dosage explanations.
\end{abstract}

\section{INTRODUCTION}

Throughout Africa, increasing resistance of Plasmodium falciparum to common antimalarials poses an important obstacle for malaria control. The introduction of artemisininbased combination therapy (ACT) has been suggested to replace ineffective treatments. ${ }^{1,2}$ However, for the successful deployment of ACT, the issue of patients' adherence needs to be considered. ${ }^{3}$ Poor levels of adherence are likely to decrease cure rates, and exposure of the parasite to subtherapeutic drug levels may favor the development of resistance. ${ }^{1}$ Measuring baseline levels of adherence and identifying possible risk factors for non-adherence are therefore important steps before the introduction of ACT. Such data should permit rational recommendations to be made that can increase the probability of correct use of the treatment. To date, there is little evidence concerning adherence to ACT in Africa. ${ }^{4,5}$

Mbarara, a town in southwestern Uganda situated in an area of mesoendemic malaria, has levels of $P$. falciparum resistance to both chloroquine (CQ) and sulfadoxinepyrimethamine (SP) greater than $30 \%{ }^{6,7}$ In the short term, if not currently, the first-line treatment for $P$. falciparum malaria in Uganda (an association of CQ and SP) is likely to be ineffective. ${ }^{8}$ Coartem ${ }^{\circledR}$ (a co-formulation of artemether and lumefantrine) has an excellent efficacy and tolerability profile, ${ }^{9-11}$ and could be a suitable alternative in Mbarara and other areas with similarly high levels of resistance, provided that the drug is taken as prescribed. However, sub-optimal adherence to artemether-lumefantrine may well represent an important obstacle to its use: the recommended method of intake is two daily doses for three days, respecting a strict eight-hour interval between the first and the second dose; also, each dose should be accompanied by a fatty meal so as to maximize lumefantrine absorption. We report a study conducted in Mbarara in 2002 with the main objectives of measuring overall adherence of patients with malaria to a six-dose artemether-lumefantrine regimen when given in an outpatient setting, and identifying risk factors for poor adherence.

\section{MATERIALS AND METHODS}

Study site and population. Mbarara District, situated in southwestern Uganda, is an area of year-round malaria transmission. Malaria is a major health problem, accounting for approximately one-third of all Outpatient Department (OPD) consultations at the regional referral hospital, Mbarara University Teaching Hospital. Plasmodium falciparum is present in more than $95 \%$ of all malaria infections. Patients resident in Mbarara Municipality, a semi-urban area with a population of 69,200 (2002 census), were screened at the hospital OPD and surrounding dispensaries. The study was reviewed and approved by the Mbarara University of Science and Technology Institutional Ethical Committee, and by the Uganda National Council of Science and Technology.

Study subjects and sample size. Patients were recruited into the study if they met the following inclusion criteria: reported fever within the last 48 hours and/or an axillary temperature $\geq 37.5^{\circ} \mathrm{C}$, a weight $\geq 10 \mathrm{~kg}$, no household member previously participating in the study, and a positive malaria blood smear containing $P$. falciparum with no signs of complicated malaria or other severe disease. To compare adherence according to differences in drug-taking behavior, patients were recruited in three age groups: $<5$ years old (young children), 5-14 years old (school age children), and $\geq 15$ years old (independent adults). Assuming an adherence of $80 \%$, a precision of $10 \%$, a type 1 error of $5 \%$ and a $20 \%$ loss to follow-up, 75 patients were needed in each age group.

Study procedures. Baseline data concerning history of the disease episode and demographic information (including the patient's address) were collected. A simple clinical examination was performed. Patients meeting inclusion criteria received an explanation that they were being offered a new drug for the treatment of malaria that was different from the antimalarials currently on the market, and were asked to provide signed written consent to receive the drug. They were given standardized instructions by a nurse on how to take the artemether-lumefantrine regimen, specifically that the second dose was to be taken eight hours after the first dose, then two 
doses per day in the morning and evening for the following two days; that all doses should be taken with food, preferably containing fat or oil; and that vomited doses should be repeated if vomiting occurred within 30 minutes of taking the dose, with the option of the patient coming to the clinic to collect a replacement dose. Patients received the latest World Health Organization (WHO) weight-specific artemetherlumefantrine (Coartem ${ }^{\circledR}$ ) blister packs (10.-14.9 kg: 1 tablet per dose; $15.0-24.9 \mathrm{~kg}$ : 2 tablets; $25.0-34.9 \mathrm{~kg}: 3$ tablets; $\geq 35$ kg: 4 tablets; Novartis Pharma AG, Basel, Switzerland). The packs contain pictorial instructions on the regimen schedule (Figure 1), and the blister is uniquely sealed so as to discourage private sale. All patients were given the first dose of artemether-lumefantrine in the clinic with milk and groundnuts and observed for 30 minutes. Patients were not aware on leaving the clinic that they were going to be visited at home or that they would be assessed for adherence. Field teams consisting of a field worker and a nurse traced the patient's home on the day after the regimen should have been completed (day 3). They introduced themselves as part of the team that had provided the patient with treatment for malaria two days earlier and asked the participants to respond to questions relating to how they took the medicine and to give blood samples for a blood smear and lumefantrine analysis. Written informed consent was then obtained from adult patients and from caregivers if the patient was less than 18 years old. A pre-piloted questionnaire was used to assess adherence. Respondents were patients themselves if $\geq 15$ years old or their caregivers if younger. An open question was provided to determine how the regimen was taken. This was followed by a structured interview concerning the time and method of taking each dose. Basic sociodemographic information was also collected (household size, level of education of respondent, number of children cared for by respondent). The blister pack was then examined, if available, for remaining tablets. A capillary blood smear for microscopic examination and a $4-\mathrm{mL}$ venous sample for quantification of blood lumefantrine levels were also collected.

Definition of adherence. Patients were classified according to the following categories of adherence, which combine findings from the blister pack check and the questionnaire. Patients who had tablets remaining in the blister pack were classified as definitely non-adherent. When the blister pack was either missing or empty, patients with information on all six doses were classified as either probably non-adherent when the patient did not report taking all doses at the correct time on the correct day and in the correct amount, or as probably adherent when the patient reported taking all doses at the correct time on the correct day and in the correct amount. Patients who vomited one or more doses were excluded from the analysis.

Laboratory methods. Slides were stained with Giemsa and read for species and parasitemia by an experienced technician. Parasitemias were calculated against 200-500 leukocytes according to the formula: parasitemia $(/ \mu \mathrm{L})=$ number of parasites $\times 8,000 /$ number of leukocytes. A slide was considered negative after 200 high-power fields had been examined. Venous blood samples were frozen at $-80^{\circ} \mathrm{C}$ and shipped to Novartis Pharma SAS Bioanalytics and Pharmacokinetics (Rueil-Malmaison, France) where lumefantrine plasma concentrations were analyzed blindly by means of a previously described high-performance liquid chromatography method ${ }^{12}$ adapted for use of mass spectrometry rather than ultraviolet detection.

Statistical analysis. Data were entered on Epi-Info version 6.04 (Centers for Disease Control and Prevention, Atlanta, GA) and analyzed using SPSS version 10.0.5 (SPSS, Inc., Chicago, IL) and Stata version 8.2 (Stata Corporation, College Station, TX) software. The three categories of adherence were presented as proportions and compared among age groups using a chi-square test. The association between adherence and several exposure variables (age, educational level of the respondent, number of children cared for by the respondent, history of antimalarial intake, presence/absence of fever on presentation) was first analyzed in a univariate model using a chi-square test. For this purpose, the two groups probably non-adherent and definitely non-adherent were combined into a non-adherent group so as to increase statistical power. Exposure variables were categorized as follows: axillary temperature as fever if $\geq 37.5^{\circ} \mathrm{C}$; parasitemia as low $(<500$ parasites $/ \mu \mathrm{L})$, medium $(500-100,000$ parasites $/ \mu \mathrm{L})$ or high $(>100,000$ parasites $/ \mu \mathrm{L})$; household size as $<5$ persons and $\geq 6$ persons; and number of children cared for by the respondent as $<2$ and $\geq 3$. Explanatory variables associated

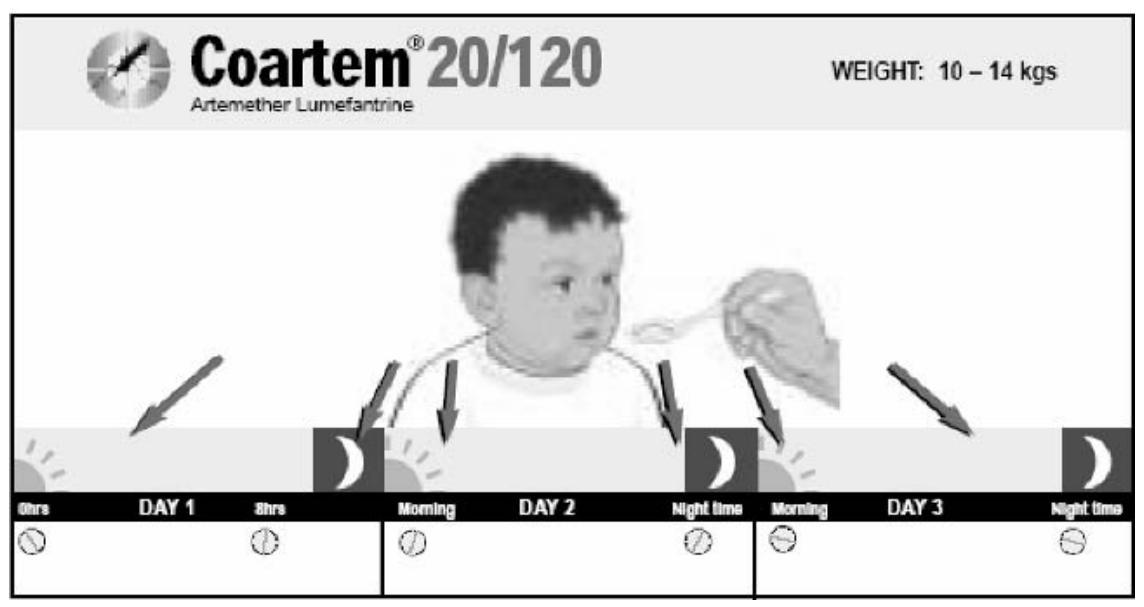

Figure 1. Pictorial instructions on the World Health Organization artemether-lumefantrine $\left(\right.$ Coartem $\left.{ }^{\circledR}\right)$ blister pack (example for weight range $=10-14 \mathrm{~kg}$ ). 
with the outcome at the $P<0.2$ level were entered in a multivariate logistic regression model. Lumefantrine plasma concentrations were described by age group and category of adherence as means with corresponding standard deviations. The association between day 3 lumefantrine plasma concentrations and the same explanatory variables as mentioned earlier was first analyzed by a univariate $t$-test adjusting for unequal variances. Variables associated with lumefantrine at the $P<0.2$ level were then entered into a multivariate analysis of variance (ANOVA) regression model and validated through Barlett's test for the equal variance assumption. Agreement (kappa statistic) between verbal responses and the tablet count provided an estimation of the reliability of the questionnaire. A kappa coefficient $>0.80$ was considered as a measure of very good reliability.

\section{RESULTS}

General characteristics of the sample. Between July and October 2002, a total of 1,277 patients were screened, of whom 235 (18.4\%) were recruited into the study. The vast majority of infections ( 225 of $235,95.7 \%$ ) were pure $P$. falciparum. Eighty-three patients were in the $<5$ years old age group, 78 in the 5-14 years old group, and 74 in the $\geq 15$ years old age group. These groups were significantly different regarding sex distribution, presence of fever at examination, and initial level of parasitemia (Table 1). Forty-four percent of patients reported taking at least one dose of an antimalarial before attending the OPD. Seventeen patients could not be traced, leaving data from 218 home interviews.

Analysis of adherence. Among the 218 patients interviewed, 8 had incomplete data for analysis of adherence due to failure to give analyzable information concerning the tim- ing of doses or the number of tablets taken per dose during the interviews. Of the remaining 210, 15 (7.1\%, 95\% confidence interval $[\mathrm{CI}]=4.0-11.5)$ had tablets remaining in the blister pack, and were thus classified as definitely nonadherent; $6(2.9 \%, 95 \% \mathrm{CI}=1.0-6.1)$ reported taking the regimen in a non-adherent manner and were thus classified as probably non-adherent; $189(90.0 \%, 95 \%$ CI $=85.1-93.7)$ reported taking the regimen in an adherent manner and were therefore classified as probably adherent. Of the 15 patients reporting missed doses, $8(53.3 \%)$ missed only one dose. The most commonly missed dose was the sixth dose $(41.7 \%$ of the missed doses). All patients but three children less than five years old (all with a parasite density $<50$ trophozoites $/ \mu \mathrm{L}$ and classified as probably adherent) had cleared their parasitemia on the day of the visit. Age group was not found to be associated with adherence (the proportions adherent in each group were $91.9 \%$ (< 5 years old), $89.0 \%$ (5-14 years old) and $88.9 \%$ ( $\geq 15$ years old) $(P=0.79)$. Four variables were significantly associated $(P<0.20)$ with being non-adherent in the univariate analysis: low parasite density $(<500$ parasites/ $\mu \mathrm{L}$ ), absence of splenomegaly, household size $<6$, and lack of formal education in the patient or the caregiver. When fitted in a multivariate model, only this last variable remained significantly associated with the outcome after allowing for potential confounders: among the 21 non-adherent patients, 6 $(28.5 \%)$ had received no formal education versus $24(12.7 \%)$ of 189 in the adherent group $(P<0.05$; odds ratio $=3.1,95 \%$ $\mathrm{CI}=1.1-9.7)$.

Analysis of plasma lumefantrine levels. Measurement of day 3 plasma lumefantrine was done on 187 patients (12 patients were traced after day 3, 2 children had failed blood draws, and 9 did not provide an analyzable sample due to small volume or accidental hemolysis), of which 16 were in

TABLE 1

Main characteristics of the study subjects

\begin{tabular}{|c|c|c|c|c|c|c|c|c|c|}
\hline & \multicolumn{8}{|c|}{ Age group (years) } & \multirow[b]{3}{*}{$P^{*}$} \\
\hline & \multicolumn{2}{|c|}{$<5$} & \multicolumn{2}{|c|}{$5-14$} & \multicolumn{2}{|c|}{15} & \multicolumn{2}{|c|}{ Total } & \\
\hline & $\mathrm{n}$ & $\%$ & $\mathrm{n}$ & $\%$ & $\mathrm{n}$ & $\%$ & $\mathrm{n}$ & $\%$ & \\
\hline \multicolumn{10}{|l|}{ Inclusion $(\mathrm{n}=235)$} \\
\hline \multicolumn{10}{|l|}{ Sex } \\
\hline Male & 50 & 60.2 & 45 & 57.7 & 26 & 45.1 & 121 & 51.5 & \multirow[t]{2}{*}{$<0.01$} \\
\hline Female & 33 & 39.8 & 33 & 42.3 & 48 & 64.9 & 114 & 48.5 & \\
\hline \multicolumn{10}{|l|}{ Fever $\left(\geq 37.5^{\circ} \mathrm{C}\right) \dagger$} \\
\hline Yes & 37 & 44.6 & 20 & 25.6 & 9 & 12.5 & 66 & 28.3 & \multirow[t]{2}{*}{$<0.01$} \\
\hline No & 46 & 55.4 & 58 & 74.4 & 63 & 87.5 & 167 & 71.7 & \\
\hline \multicolumn{10}{|l|}{ Parasitemia $(/ \mu \mathrm{L})$} \\
\hline$<500$ & 18 & 21.7 & 22 & 28.9 & 29 & 39.7 & 69 & 30.0 & \multirow[t]{3}{*}{$<0.01$} \\
\hline $500-100,000$ & 54 & 65.1 & 47 & 61.8 & 44 & 60.3 & 143 & 62.0 & \\
\hline$>100,000$ & 11 & 13.2 & 7 & 9.2 & 0 & 0.0 & 18 & 8.0 & \\
\hline \multicolumn{10}{|c|}{ House visit $(\mathrm{n}=218)$} \\
\hline \multicolumn{10}{|c|}{$\begin{array}{l}\text { Formal education of } \\
\text { patient or caregiver }\end{array}$} \\
\hline None & 13 & 16.5 & 14 & 18.7 & 3 & 4.7 & 30 & 13.7 & \multirow[t]{2}{*}{0.04} \\
\hline At least primary & 66 & 83.5 & 61 & 81.3 & 61 & 95.3 & 188 & 86.3 & \\
\hline \multicolumn{10}{|l|}{ Household size (n) } \\
\hline $0-5$ & 59 & 74.7 & 43 & 57.3 & 50 & 78.1 & 152 & 69.7 & \multirow[t]{2}{*}{0.01} \\
\hline$\geq 6$ & 20 & 25.3 & 32 & 42.7 & 14 & 21.9 & 66 & 30.3 & \\
\hline \multicolumn{10}{|c|}{$\begin{array}{l}\text { Children cared for by } \\
\text { respondent }(n)\end{array}$} \\
\hline $0-2$ & 61 & 77.2 & 58 & 77.3 & 60 & 93.8 & 179 & 82.1 & \multirow[t]{2}{*}{0.01} \\
\hline$\geq 3$ & 18 & 22.8 & 17 & 22.7 & 4 & 6.3 & 39 & 17.9 & \\
\hline
\end{tabular}

* Chi-square for the difference in proportions between the three age groups, for the corresponding variable.

$\dagger$ Two missing values for fever. 
the non-adherent group and 171 in the adherent group. The median lumefantrine level was $2.45 \mu \mathrm{g} / \mathrm{mL}$ (range $=$ 0.06-11.4), with median levels in those less than five years old significantly lower than in the two other age groups $(P<0.01)$ (Figure 2). Wide variations in individual levels were seen in each category of age. Mean lumefantrine levels were lower in the non-adherent group $(2.76 \mu \mathrm{g} / \mathrm{mL}, 95 \% \mathrm{CI}=1.06-4.45)$ than in the adherent group $(3.23 \mu \mathrm{g} / \mathrm{mL}, 95 \%$ CI $=$ 2.87-3.59), but this difference was not statistically significant $(P=0.46)$. Six variables were significantly associated with a low plasma lumefantrine concentration after univariate analysis: an age less than 5 years $(P<0.01)$, pallor $(P=0.04)$, splenomegaly $(P=0.19)$, abdominal pain $(P<0.01)$, a history of jaundice $(P=0.02)$, and a lack of formal education $(P<$ 0.01). After multivariate ANOVA regression, four variables remained significantly associated with lumefantrine level: lack of formal education, an age less than 5 years, and history of jaundice were associated with lower plasma lumefantrine, whereas abdominal pain was associated with higher lumefantrine levels (Table 2).

Validation of the questionnaire. Of the 218 patients visited, $182(83.5 \%)$ had the blister pack available. Of the responses, $97.3 \%$ (177 of 182) were concordant (i.e., the patient reported finishing the treatment course and there were no tablets left in the blister pack, or the patient reported not finishing the treatment and there were tablets remaining in the blister pack). The remaining $2.7 \%$ (5 of 182) of responses were discordant. The kappa coefficient was thus 0.81 (95\% CI = 0.54-0.95), thus showing very good agreement between these two complementary methods of assessing adherence.

\section{DISCUSSION}

The high levels of adherence demonstrated in this study are very encouraging with regard to the feasibility of widespread
TABLE 2

Multivariate analysis of variance regression model of the association of several exposure variables with day 3 plasma lumefantrine concentration

\begin{tabular}{lrcc}
\hline \multicolumn{1}{c}{ Variable } & \multicolumn{1}{c}{$P$} & Coefficient & 95\% confidence interval \\
\hline No formal education & 0.03 & -1.01 & $-1.90--0.12$ \\
Age $<$ 5 years & $<0.01$ & -1.46 & $-2.14-0.78$ \\
History of jaundice & $<0.01$ & -2.67 & $-4.26--1.08$ \\
Abdominal pain & 0.01 & 0.86 & $0.21-1.51$ \\
\hline
\end{tabular}

introduction of artemether-lumefantrine. Only 7.1\% (95\% $\mathrm{CI}=4.0-11.5)$ of patients were definitely non-adherent, a figure lower than that found in two other similar studies conducted in Zambia $^{4}$ and southern Sudan, ${ }^{5}$ where $18.3 \%$ and $21.2 \%$ of the patients were definitely non-adherent to a threeday regimen of artesunate plus SP and artemether-lumefantrine, respectively. However, these latter studies were conducted in complex settings (refugee and internally displaced populations), which are not easily comparable to our stable site.

Several factors may have contributed to these high levels of adherence. The drug used was the latest WHO-approved blister pack, which contains pictorial instructions on how to take the drug, a factor that has been shown to improve adherence. ${ }^{13}$ A detailed verbal explanation was also given with the drug with clear instructions on how to take the medication, an intervention shown to contribute to improved adherence. ${ }^{14}$ The rapid clinical improvement under ACT treatment ${ }^{15}$ may also have encouraged patients to complete the regimen, although, conversely, feeling better may have been a reason for some patients to miss doses on the last day, as was the case in almost half of the missed doses in our study. The lack of a relationship between adherence and age group in Mbarara was an important finding, demonstrating that tablets were

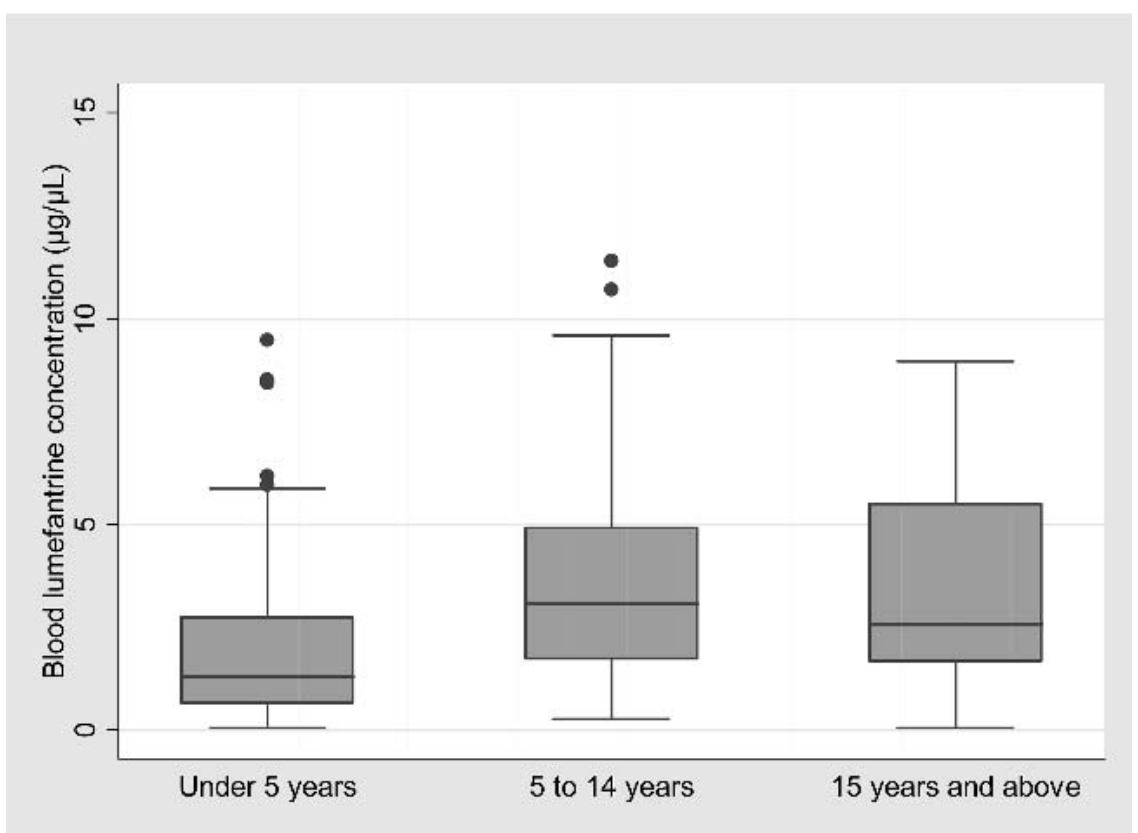

FigurE 2. Box plots of blood lumefantrine concentrations by age group. Error bars represent the first (lower bar) and third (upper bar) quartiles. The horizontal lines in the boxes represent the medians. 
well accepted by young children, and that the standard instructions given were suitable both for patients and for caregivers.

An important finding of our study was the association between education of the patient/caregiver and adherence, which mirrored results from Zambia. ${ }^{4}$ Education may have an effect on the patient/caregiver understanding the instructions given in the clinic, the quality of the patients' relationship with the health care provider, and the ability of the patient to interpret pictorial instructions on the artemether-lumefantrine pack. However, educational attainment is also a proxy measure for other sociodemographic and economic factors that may affect adherence, and which were not investigated in this study. The generally high educational level of the semiurban population in Mbarara indicates that in more rural and/ or less educated populations, extra emphasis on appropriate health education messages would increase the quality of counseling and promote high levels of adherence. ${ }^{16,17}$

The main results of the lumefantrine analysis confirm what has already been described in the literature, although these findings may not always have a very clear explanation. ${ }^{9,18}$ First, lumefantrine levels seem to be lower in very young children, which probably reflects differences in absorption and metabolism. Second, lumefantrine levels on the day after completion of treatment vary largely from one individual to another, even for patients of similar age groups. In our site, this may have been due to differences in fat intake, one of the major determinants of lumefantrine absorption, which was not measured in our study. The fraction of the dose absorbed is highly variable between patients, and also between doses. ${ }^{18}$ Lumefantrine levels seven days after the first dose have been shown to be more closely related to cure rates, and therefore probably to adherence. We did not re-sample patients on day 7 because the day 3 questionnaire could have encouraged non-adherent respondents to complete the regimen, thus introducing a bias. In our study, there was some evidence that non-adherence caused lower day 3 lumefantrine levels, although this association was not statistically significant, probably due to insufficient power. We had hypothesized that plasma lumefantrine levels on day 3 would show a correlation with adherence, i.e., plasma levels would be lower in nonadherent than in adherent patients. This rational was based on data showing that the maximum plasma lumefantrine concentrations were reached mainly on day 3 after the last dose of a six-dose regimen given to malaria patients. ${ }^{9,18,19}$ We assumed, therefore, that lumefantrine levels on day 3 would be lower, on average, in patients who had failed to take the complete six doses regimen. Our data proved this to be the case, although due to the low number of patients in the nonadherent group, this difference was not statistically significant. Further studies including more patients may be necessary to confirm that lumefantrine plasma levels on day 3 can be used as a surrogative marker of adherence. Young age was associated with low lumefantrine levels on day 3 , an observation which, as mentioned earlier, may reflect differences in absorption and metabolism. Lack of education was also associated with lower lumefantrine, perhaps an indirect result of lower adherence. The association between decreased lumefantrine plasma concentrations and jaundice could be due to a reduction of the bioavailability of the drug in patients with decreased bile production, as has been observed in animal models (see Discussion section on Ezzet and others ${ }^{19}$ ). The association between increased lumefantrine plasma concentrations and abdominal pain is less clear and could be merely be due to chance. However abdominal pain is a known side effect associated with the artemether-lumefantrine drug combination, ${ }^{9}$ and suggests that elevated plasma lumefantrine levels account for this side effect.

The high levels of adherence to artemether-lumefantrine found in our study suggest that this drug, given in its current WHO formulation, is likely to be very effective in Mbarara and in other regions with high levels of resistance to other antimalarials, provided that clear explanations on how to take the treatment are given to the patient. Particular attention should be paid to people with a low level of formal education. The investment to improve patient adherence should be integral to the introduction of combination therapy. Considering issues of adherence whilst introducing ACTs, and monitoring adherence after they are deployed, are essential to help ensure long-term treatment efficacy.

Received February 27, 2004. Accepted for publication April 29, 2004.

Acknowledgments: We thank all the staff who participated in the study. We also thank the staff and officials of Mbarara University Teaching Hospital, Mbarara University of Science and Technology, and the Kakoba Dispensary for their cooperation. We are very grateful to Loretxu Pinoges and Evelyn Depoortere (Epicentre) for their helpful advice on data analysis. Novartis Pharma AG performed free pharmacokinetic analyses, but did not take part in study design or analysis. We thank Marie-Noëlle Bizot (Novartis Pharma AG) for performing the lumefantrine measurements.

Financial support: This study was funded by Médecins sans Frontières.

Authors' addresses: Carole Fogg, Patrice Piola, and Samuel Biraro, Epicentre, PO Box, Kampala, Uganda, Telephone/Fax: 256-41-269998. Francis Bajunirwe and James Kiguli, Mbarara University of Science and Technology, PO Box 1410, Mbarara, Uganda, Telephone: 256-77-576-396, Fax: 256-48-520-782. Francesco Checchi and JeanPaul Guthmann, Epicentre, 42, Boulevard Richard Lenoir, 75011 Paris, France, Telephone: 33-1-40-21-28-48, Fax: 33-1-40-21-28- 03. Proscovia Namiiro, Joy Musabe, and Agnes Kyomugisha, Mbarara University Teaching Hospital, PO Box 1410, Mbarara, Uganda, Telephone: 256-48-520-332, Fax: 256-48-520-782.

Reprint requests: Jean-Paul Guthmann, Epicentre, 8 Rue SaintSabin, 75011 Paris, France, Telephone: 33-1-40-21-28-48, Fax: 33-140-21-28-03, E-mail: jguthmann@epicentre.msf.org.

\section{REFERENCES}

1. White NJ, Olliaro P, 1996. Strategies for the prevention of antimalarial drug resistance: rationale for combination chemotherapy for malaria. Parasitol Today 12: 399-341.

2. White NJ, 1999. Delaying antimalarial drug resistance with combination chemotherapy. Parassitologia 41: 301-308.

3. Bloland PB, Ettling M, Meek S, 2000. Combination therapy for malaria in Africa: hype or hope? Bull World Health Organ 78: 1378-1388.

4. Depoortere E, Guthmann JP, Sipilnyambe N, Nkandu E, Fermon F, Balkan S, Legros D, 2004. Adherence to the combination of sulfadoxine-pyrimethamine and artesunate in the Maheba Refugee Settlement, Zambia. Trop Med Int Health 9: 1-6.

5. Depoortere E, Salvador ET, Stivanello E, Bisoffi Z, Guthmann JP. Adherence to a combination of artemether and lumefantrine $\left(\right.$ Coartem $\left.^{\circledR}\right)$ in Kajo Keji, Southern Sudan. Ann Trop Med Parasitol 98: 635-637.

6. Legros D, Johnson K, Houpikan P, Makanga M, Kabakyenga JK, Talisuna AO, Taylor WR, 2001. Clinical efficacy of chloroquine or sulfadoxine-pyrimethamine in children under five from south-western Uganda with uncomplicated falciparum malaria. Trans R Soc Trop Med Hyg 96: 199-201. 
7. Priotto G, Kabakyenga J, Pinoges L, Ruiz A, Erikson T, Coussement F, Ngambe T, Taylor WRJ, Perea W, Guthmann JP, Olliaro P, Legros D, 2003. Artesunate and sulfadoxinepyrimethamine combinations for the treatment of uncomplicated falciparum malaria in Uganda: a randomised, doubleblind, placebo-controlled trial. Trans R Soc Trop Med Hyg 97: 325-330.

8. Kamya MR, Bakyaita NN, Talisuna AO, Were WM, Staedke SG, 2002. Increasing antimalarial drug resistance in Uganda and revision of the national drug policy. Trop Med Int Health 7: 1031-1041.

9. Lefèvre G, Looareesuwan S, Treeprasertsuk S, Krudsood S, Silachamroon U, Gathmann I, Mull R, Bakshi R, 2001. A clinical and pharmacokinetic trial of six doses of artemetherlumefantrine for multidrug-resistant Plasmodium falciparum malaria in Thailand. Am J Trop Med Hyg 64: 247-256.

10. van Vugt M, Ezzet F., Nosten F, Gathmann I, Wilairatana P, Looareesuwan S, White NJ, 1999. Efficacy of six doses of artemether-lumefantrine (benflumetol) in multidrug resistant Plasmodium falciparum malaria. Am J Trop Med Hyg 60: 936-942.

11. van Vugt M, Looareesuwan S, Wilairatana P, McGready R, Villegas L, Gathmann I, Mull R, Brockman A, White NJ, Nosten F, 2000. Artemether-lumefantrine for the treatment of multidrug-resistant falciparum malaria. Trans $R$ Soc Trop Med Hyg 94: 545-548.

12. Mansor SM, Navaratnam V, Yahaya N, Nair NK, Wernsdorfer WH, Degen PH, 1996. Determination of a new antimalarial drug, benflumetol, in blood plasma by high-performance liquid chromatography. J Chromatogr B Biomed Sci Appl 682: 321325.

13. Qingjun L, Jihui D, Laiyi T, Xiangjun Z, Jun L, Hay A, Shires S, Navaratnam V, 1998. The effect of drug packaging on patients' adherence with treatment for Plasmodium vivax malaria in China. Bull World Health Organ 76: 21-27.

14. Okonkwo PO, Akpala CO, Okafor HU, Mbah AU, Nwaiwu O, 2001. Compliance to correct dose of chloroquine in uncomplicated malaria correlates with improvement in the condition of rural Nigerian children. Trans R Soc Trop Med Hyg 95: 320324.

15. Nosten F, Brasseur P, 2002. Combination therapy for malaria: the way forward? Drugs 62: 1315-1329.

16. Denis MB, 1998. Improving adherence with quinine + tetracycline for treatment of malaria: evaluation of health education interventions in Cambodian villages. Bull World Health Organ 76: 43-49.

17. Slutsker L, Chitsulo L, Macheso A, Steketee RW, 1994. Treatment of malaria fever episodes among children in Malawi: results of a KAP survey. Trop Med Parasitol 45: 61-64.

18. White NJ, van Vugt M, Ezzet F, 1999. Clinical pharmacokinetics and pharmacodynamics of artemether-lumefantrine. Clin Pharmacokinet 37: 105-125.

19. Ezzet F, van Vugt M, Nosten F, Looareesuwan S, White NJ, 2000. Pharmacokinetics and pharmacodynamics of lumefantrine (benflumetol) in acute falciparum malaria. Antimicrobial Agents Chemother 44: 697-704. 